\title{
Newton iteration acceleration of the Nash game algorithm for power control in 3G wireless CDMA networks *
}

\author{
Zoran Gajic ${ }^{a}$ and Sarah Koskie ${ }^{b}$ \\ ${ }^{a}$ Electrical \& Comp. Eng. Dept., Rutgers Univ., 94 Brett Road, Piscataway, NJ 08854-8058 \\ ${ }^{b}$ Electrical \& Comp. Eng. Dept., IUPUI, 723 W. Michigan St., Indianapolis IN 46202
}

\begin{abstract}
In wireless communication systems, each user's signal contributes to the interference seen by the other users. Given limited available battery power, this creates a need for effective and efficient power control strategies. These strategies may be designed to achieve quality of service (QoS) or system capacity objectives, or both. We show how the power control problem is naturally suited to formulation as a noncooperative game in which users choose to trade off between signal-to-interference ratio (SIR) error and power usage. Koskie (2003) studied the static Nash game formulation of this problem. The solution obtained led to a system of nonlinear algebraic equations. In this paper we present a novel distributed power control strategy based on the Newton iteration used to solve the corresponding algebraic equations. That method accelerates the convergence of the Nash game algorithm owing to the quadratic convergence of the Newton iterations. A numerical example demonstrates the efficiency of the new algorithm.
\end{abstract}

Keywords: wireless networks, power control, Nash equilibrium, Newton iterations

\section{INTRODUCTION}

Closed loop power control is used in wireless communication networks to compensate for fast fading and timevarying channel characteristics, as well as to reduce mobile battery power consumption.

One of the most common approaches to closed-loop power control in wireless communication networks is SIR balancing, also called power balancing. The SIR balancing solution was originally derived for satellite communications by Aein ${ }^{1}$ and Meyerhoff, ${ }^{2}$ and adapted for wireless communications by Nettleton ${ }^{3}$ and Zander. ${ }^{4,5}$ SIR balancing algorithms are simple and most can be implemented distributively, but have the disadvantage that convergence can be slow and is guaranteed only if every mobile's target SIR is feasible.

An alternative framework for developing power control algorithms is based on game theory or economic formulations requiring the specification of a utility or cost function. ${ }^{6-8}$ In Koskie, ${ }^{9}$ a power control algorithm using fixed point iterations was proposed for solving the system of nonlinear equations specifying the Nash equilibrium solution to the problem.

We consider the uplink direction for a single cell CDMA system with $N$ users, designating the transmitted power and SIR for the $i$ th user by $p_{i}$ and $\gamma_{i}$, respectively. We denote the background (receiver) noise power within the user's bandwidth by $\eta_{i}:=\sigma_{i}^{2}$. The interference experienced by the $i$ th user will be designated $I_{i}\left(p_{-i}\right)$ where the subscript " $i$ " indicates that the interference depends on the powers of all users except the $i$ th. We use a "snapshot" model, assuming that link gains evolve slowly with respect to SIR evolution. In this problem formulation, the SIR of mobile $i$ is given by

$$
\gamma_{i}=\frac{g_{i i} p_{i}}{I_{i}\left(p_{-i}\right)}=\frac{g_{i i} p_{i}}{\sum_{j \neq i} g_{i j} p_{j}+\eta_{i}}
$$

with the interference defined by

$$
I_{i}\left(p_{-i}\right):=\sum_{j \neq i} g_{i j} p_{j}+\eta_{i}
$$

where the elements $g_{i j}$ of the gain matrix $\mathbf{G}$ are derived below. In the deterministic formulation of the power control problem for wireless networks, the noise power $\eta_{i}$ is treated as constant.

This work was supported by National Science Foundation grant ANIR-0106857. 
The elements $g_{i j}$ of the gain matrix $\mathbf{G}$ depend on mobile-to-base station distance, physical parameters, and code correlation coefficients. For the uplink, the SIR of the $i$ th mobile is determined by

$$
\gamma_{i}=\frac{h_{i} p_{i}}{\sum_{j \neq i} h_{j} p_{j} c_{i j}+\eta_{i}}
$$

where $h_{i}$ is the attenuation from the $i$ th mobile to the base station and $c_{i j}$ is the code correlation coefficient. The attenuation is calculated from the distance $r_{i}$ between the mobile and base station to be $h_{i}=A / r_{i}^{\alpha}$ in the absence of shadow and fast fading. $A$ is a constant gain and the coefficient $\alpha$ is usually in the range of 3 to 4 for outdoor communications. The code correlation coefficient $c_{i j}$ is computed from the signatures $\mathbf{S}_{\mathbf{i}}$ and $\mathbf{s}_{\mathbf{j}}$ to be $c_{i j}=\left(\mathbf{s}_{\mathbf{j}}^{T} \mathbf{s}_{\mathbf{i}}\right)^{2}$. Putting these together we find that the gain matrix $\mathbf{G}$ for the uplink has elements

$$
g_{i j}:= \begin{cases}h_{i} & j=i \\ h_{j}\left(\mathbf{s}_{\mathbf{j}}^{T} \mathbf{s}_{\mathbf{i}}\right)^{2} & \text { otherwise. }\end{cases}
$$

\section{NASH GAME PROBLEM FORMULATION}

In this section we formulate the SIR-based power control problem as a noncooperative game, choose an appropriate cost function, and find the corresponding Nash equilibrium ${ }^{10,11}$ power vector.

To the $i$ th user, we assign the cost function $J_{i}\left(p_{i}, \gamma_{i}(\mathbf{p})\right)$ where the power vector is $\mathbf{p}:=\left[p_{1}, p_{2}, \ldots, p_{N}\right]^{T}$. The Nash equilibrium strategies corresponding to these costs are the power vectors $\mathbf{p}^{*}$ having the property that no individual user can lower its cost by deviating from $p_{i}^{*}$. In other words, $\mathbf{p}^{*}$ satisfies

$$
J_{i}\left(p_{i}^{*}, \gamma_{i}\left(\mathbf{p}^{*}\right)\right) \leq J_{i}\left(p_{i}, \gamma_{i}\left(p_{1}^{*}, p_{2}^{*}, \ldots, p_{i-1}^{*}, p_{i}, p_{i+1}^{*}, \ldots, p_{N}^{*}\right)\right), \quad \forall p_{i}, \quad \forall i=1,2, \ldots, N .
$$

In general, a cost function should be convex and positive. Power always being positive in this application, a sensible choice for our cost function is

$$
J_{i}\left(p_{i}, \gamma_{i}\right)=b_{i} p_{i}+c_{i}\left(\gamma_{i}^{\text {tar }}-\gamma_{i}\right)^{2},
$$

$b_{i}$ and $c_{i}$ being constant weighting factors. We will see in the analysis below that only the ratio of the power weight $b_{i}$ to the SIR-error weight $c_{i}$ is important; hence, the $c_{i}$ can be made equal to one by replacing $b_{i}$ by $\tilde{b}_{i}=b_{i} / c_{i}$. For different applications, different ratios $b_{i} / c_{i}$ may be chosen. Choosing $b_{i} / c_{i}>1$ places more emphasis on power usage whereas $b_{i} / c_{i}<1$ places more emphasis on SIR error.

Applying the necessary conditions for a Nash equilibrium we have

$$
\frac{\partial J_{i}}{\partial p_{i}}=0=b_{i}-2 c_{i}\left(\gamma_{i}^{t a r}-\gamma_{i}\right) \frac{\partial \gamma_{i}}{\partial p_{i}}=b_{i}-2 c_{i}\left(\gamma_{i}^{t a r}-\gamma_{i}\right) \frac{g_{i i}}{\sum_{j \neq i} g_{i j} p_{j}+\eta_{i}} .
$$

Recalling that $I_{i}\left(p_{-i}\right):=\sum_{j \neq i} g_{i j} p_{j}+\eta_{i}$, and rearranging terms yields

$$
\gamma_{i}=\gamma_{i}^{t a r}-\frac{b_{i} I_{i}\left(p_{-i}\right)}{2 c_{i} g_{i i}}
$$

It follows from (8) that as $b_{i} \rightarrow 0$ (power expenditure ceases to be important) that $\gamma_{i} \rightarrow \gamma_{i}^{\text {tar }}$. Likewise, as $c_{i} \rightarrow 0$, (SIR value of no importance), $\gamma_{i}$ may diverge widely from the large value $\gamma_{i}^{t a r}$. In this latter case the only possible way to achieve high SIR's would be to set $\gamma_{i}^{t a r}$ as high as possible, which is not practical. Substituting for $\gamma_{i}$ from (1) and isolating $p_{i}$, we can express the required power in terms of given and measured quantities as

$$
p_{i}=\frac{\gamma_{i}^{t a r}}{g_{i i}} I_{i}\left(p_{-i}\right)-\frac{b_{i} I_{i}^{2}\left(p_{-i}\right)}{2 c_{i} g_{i i}^{2}}
$$

Substituting for the interference using (2) in (8), and evaluating at the Nash equilibrium we have

$$
\gamma_{i}^{*}=\left\{\begin{array}{cl}
\gamma_{i}^{t a r}-\frac{b_{i}}{2 c_{i} g_{i i}}\left(\frac{g_{i i} p_{i}^{*}}{\gamma_{i}^{*}}\right) & \text { if this quantity is nonnegative } \\
0 & \text { otherwise. }
\end{array}\right.
$$


Of course the equilibrium power corresponding to zero SIR is $p_{i}^{*}=0$. Otherwise (10) yields an expression for the Nash equilibrium power $p_{i}^{*}$ in terms of the cost weighting coefficients, the target SIR, and the Nash equilibrium SIR $\gamma_{i}^{*}$, namely

$$
p_{i}^{*}=\frac{2 c_{i}}{b_{i}} \gamma_{i}^{*}\left(\gamma_{i}^{t a r}-\gamma_{i}^{*}\right) .
$$

As expected, the Nash (noncooperative) equilibrium has SIR $\gamma_{i}^{*}$ less than $\gamma_{i}^{t a r}$. When mobiles cooperate, as they must in the application of the power balancing algorithm, the target SIR, if feasible, will be attained by all mobiles.

\section{FIXED-POINT ALGORITHM FOR POWER UPDATES}

We assume that the algorithm will run in real time using measurements that are potentially updated at every step of the algorithm. The fixed point iterations for solving (9) can be expressed in the form $p_{i}^{(k+1)}=f_{i}^{(k)}\left(p_{i}^{(k)}\right)$ as

$$
p_{i}^{(k+1)}=\frac{\gamma_{i}^{\text {tar }}}{g_{i i}} I_{i}^{(k)}-\frac{b_{i}}{2 c_{i}}\left(\frac{I_{i}^{(k)}}{g_{i i}}\right)^{2}=: f_{i}\left(p_{i}^{(k)}\right),
$$

where $p_{i}^{(k)}$ is the power of the $i$ th mobile and $I_{i}^{(k)}$ the measured interference experienced by the $i$ th mobile at the $k$ th step of the algorithm and we recall that $I_{i}^{(k)}=\sum_{j \neq i} g_{i j} p_{j}^{(k)}+\eta_{i}$. Of course the mobile power cannot be negative, so there is an implicit assumption here that whenever this expression is negative, the assigned power will be zero.

For computational efficiency, we may rewrite the algorithm in terms of the previous power value $p_{i}^{(k)}$ and current SIR measurement $\gamma_{i}^{(k)}$ by

$$
p_{i}^{(k+1)}=\gamma_{i}^{\operatorname{tar}}\left(\frac{p_{i}^{(k)}}{\gamma_{i}^{(k)}}\right)-\frac{b_{i}}{2 c_{i}}\left(\frac{p_{i}^{(k)}}{\gamma_{i}^{(k)}}\right)^{2}
$$

where we have substituted for $I_{i}^{(k)}$ in (12) using $\gamma_{i}^{(k)}=g_{i i} p_{i}^{(k)} / I_{i}^{(k)}$. Note that algorithm (13) differs from the power balancing algorithm in that, to the linear (in power) term, a quadratic (in power) term is added. As before, the power is required to be nonnegative.

Both formulations of the algorithm require only a single measurement at each step. Accordingly, if this measurement is made available to the mobile, either algorithm can be used to implement a distributed power control. One minor difference between the two formulations of the algorithm is that the formulation in terms of power (13), like the power balancing algorithm, requires nonzero initial powers. The formulation in terms of interference, however, does not require an initial nonzero power because the interference, which includes the noise power, is never zero.

\section{ACCELERATED ALGORITHM USING NEWTON ITERATIONS}

The Newton iterations are obtained by replacing the quadratic term of the fixed-point iteration (12) by a linear approximation. ${ }^{12,13}$ We define $\Delta_{I_{i}}:=I_{i}^{(k+1)}-I_{i}^{(k)}$. Then

$$
\begin{aligned}
\left(I_{i}^{(k+1)}\right)^{2} & =I_{i}^{(k+1)}\left(I_{i}^{(k)}+\Delta_{I_{i}}\right) \\
& =I_{i}^{(k+1)} I_{i}^{(k)}+\left(I_{i}^{(k)}+\Delta_{I_{i}}\right) \Delta_{I_{i}} \\
& =I_{i}^{(k+1)} I_{i}^{(k)}+I_{i}^{(k)}\left(I_{i}^{(k+1)}-I_{i}^{(k)}\right)+\left(\Delta_{I_{i}}\right)^{2} \\
& \simeq 2 I_{i}^{(k+1)} I_{i}^{(k)}-\left(I_{i}^{(k)}\right)^{2} .
\end{aligned}
$$


Substituting into the quadratic term of (12) we obtain

$$
\begin{aligned}
p_{i}^{(k+1)} & =\frac{\gamma_{i}^{\text {tar }}}{g_{i i}} I_{i}^{(k+1)}-\frac{b_{i}}{2 c_{i}}\left(\frac{I_{i}^{(k+1)}}{g_{i i}}\right)^{2} \\
& \simeq \frac{\gamma_{i}^{t a r}}{g_{i i}} I_{i}^{(k+1)}-\frac{b_{i}}{2 c_{i} g_{i i}^{2}}\left(2 I_{i}^{(k+1)} I_{i}^{(k)}-\left(I_{i}^{(k)}\right)^{2}\right) \\
& =\left(\frac{\gamma_{i}^{t a r}}{g_{i i}}-\frac{b_{i}}{c_{i} g_{i i}^{2}} I_{i}^{(k)}\right) I_{i}^{(k+1)}+\frac{b_{i}}{2 c_{i} g_{i i}^{2}}\left(I_{i}^{(k)}\right)^{2} .
\end{aligned}
$$

Since

$$
I_{i}^{(k+1)}=\sum_{j \neq i} g_{i j} p_{i j}^{(k+1)}+\eta_{i}
$$

the power update equation becomes

$$
p_{i}^{(k+1)}-\alpha_{i}^{(k)} \sum_{j \neq i} g_{i j} p_{i j}^{(k+1)}=\beta_{i}^{(k)}+\alpha_{i}^{(k)} \eta_{i},
$$

where we have defined the variables

$$
\alpha_{i}^{(k)}:=\frac{1}{g_{i i}}\left(\gamma_{i}^{\text {tar }}-\frac{b_{i}}{c_{i} g_{i i}} I_{i}^{(k)}\right)
$$

and

$$
\beta_{i}^{(k)}:=\frac{b_{i}}{2 c_{i} g_{i i}^{2}}\left(I_{i}^{(k)}\right)^{2}
$$

to simplify the expression. (22) defines a system of linear equations which can be expressed as

$$
\mathbf{A}\left(\mathbf{p}^{(k)}\right) \mathbf{p}^{(k+1)}=\mathbf{b}\left(\mathbf{p}^{(k)}\right)
$$

where the $i, j$ th element of the $\mathbf{A}\left(\mathbf{p}^{(k)}\right)$ matrix is

$$
a_{i j}^{(k)}=\left\{\begin{array}{cl}
1 & \text { if } i=j \\
-\alpha_{i}^{(k)} g_{i j} & \text { otherwise }
\end{array}\right.
$$

and the $i$ th element of the $\mathbf{b}\left(\mathbf{p}^{(k)}\right)$ vector is

$$
b_{i}^{(k)}=\beta_{i}^{(k)}+\alpha_{i}^{(k)}
$$

Note that the Newton iterations converge quadratically so long as a good enough initial guess is provided. For our purposes, a small positive value has been found to be acceptable.

\section{EXPERIMENTAL RESULTS}

We ran a MATLAB simulation to test the above update equations. For a three-mobile system, using the gain matrix

$$
G=\left[\begin{array}{lll}
1 & 0.0882 & 0.0357 \\
0.1524 & 0.9500 & 0.3501 \\
0.0670 & 0.0244 & 0.9900
\end{array}\right]
$$

and initial powers values all equal to 0.1 , we obtained very fast convergence to the Nash equilibrium. Noting the different scales on the horizontal axes, it can be seen in Figures 1 and 2, that the Newton iterations converge much faster (quadratically) than the fixed-point iterations (which have linear convergence).

The SIR's for the two algorithms are shown in Figures 3 and 4. 


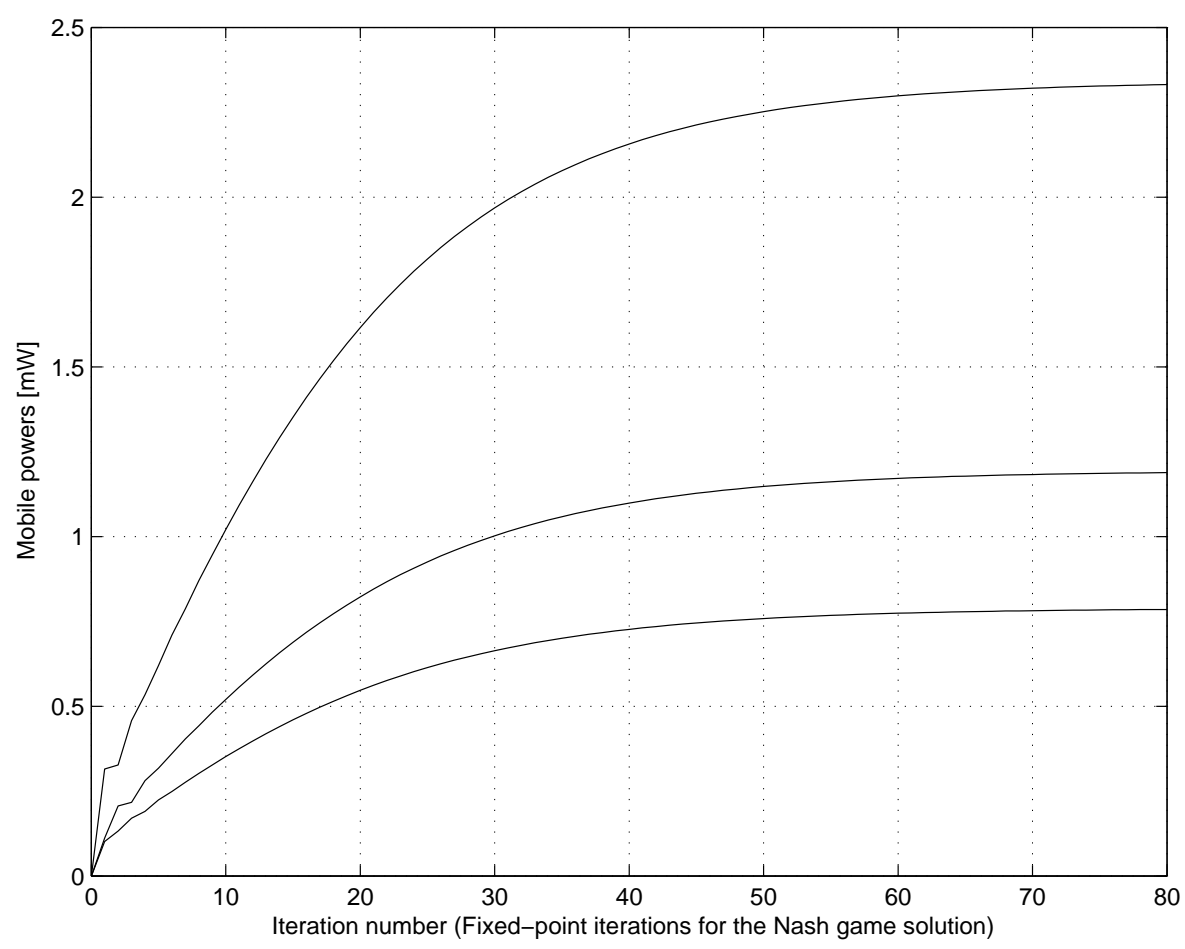

Figure 1. Powers computed for the three mobiles using fixed-point iterations.

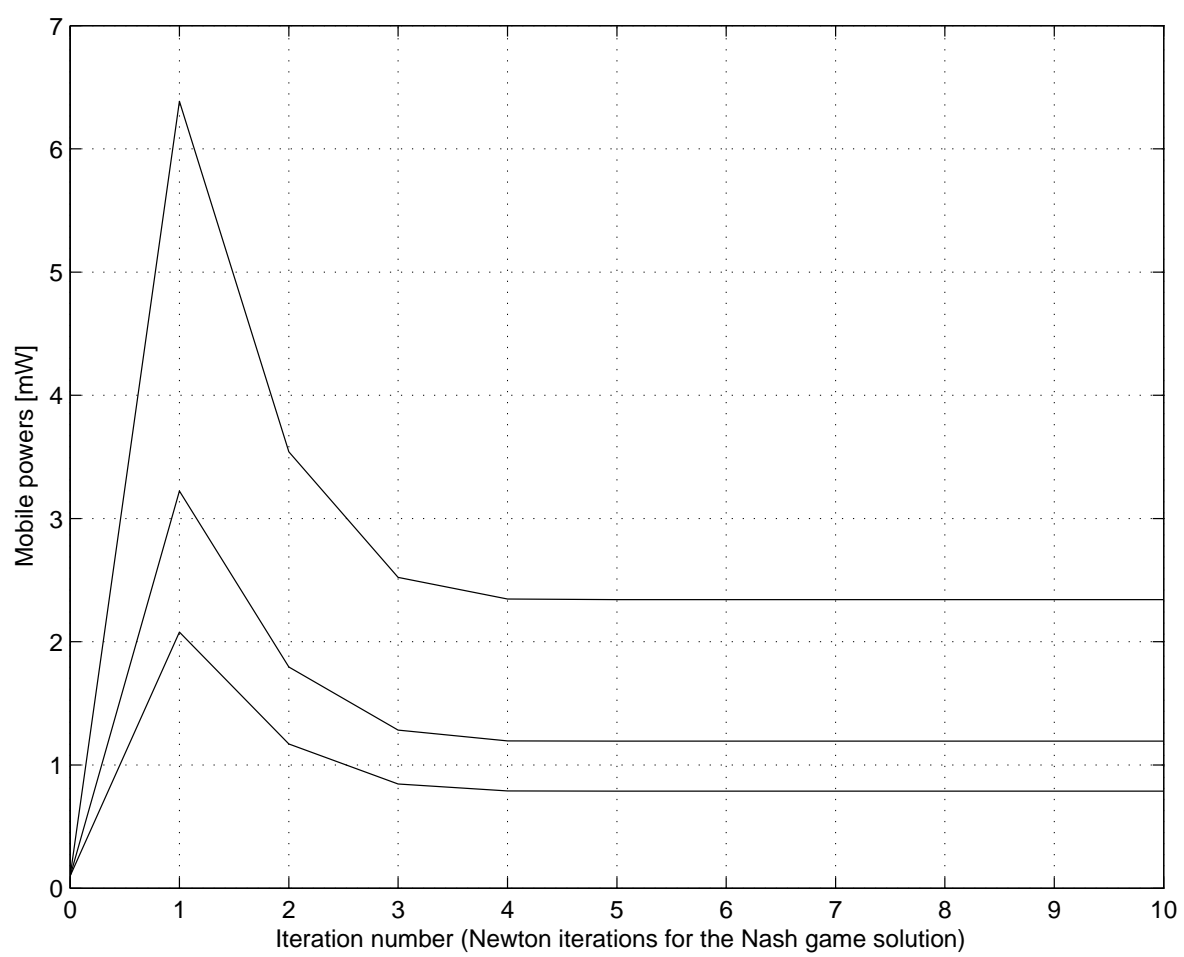

Figure 2. Powers computed for the three mobiles using fixed-point iterations. 


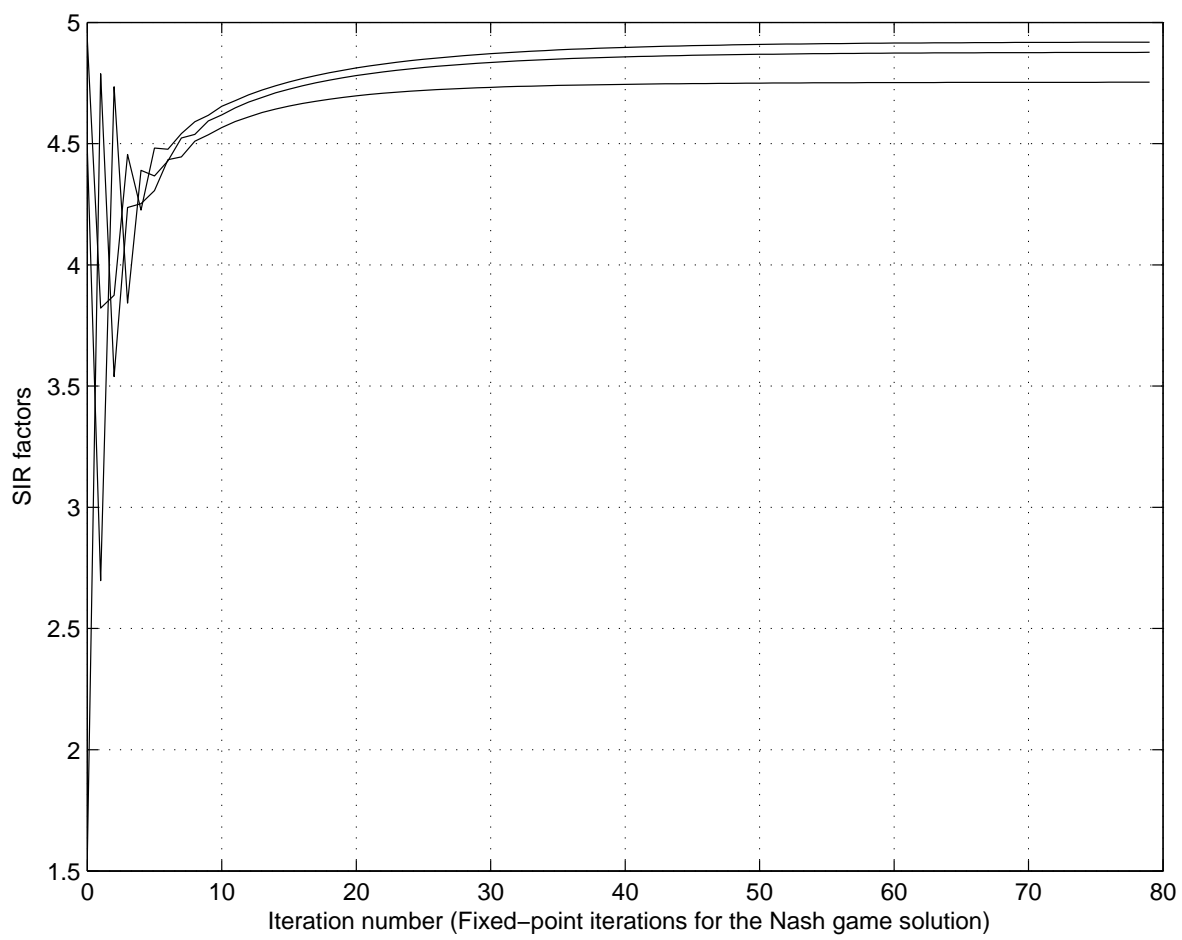

Figure 3. SIR's computed for the three mobiles using fixed-point iterations.

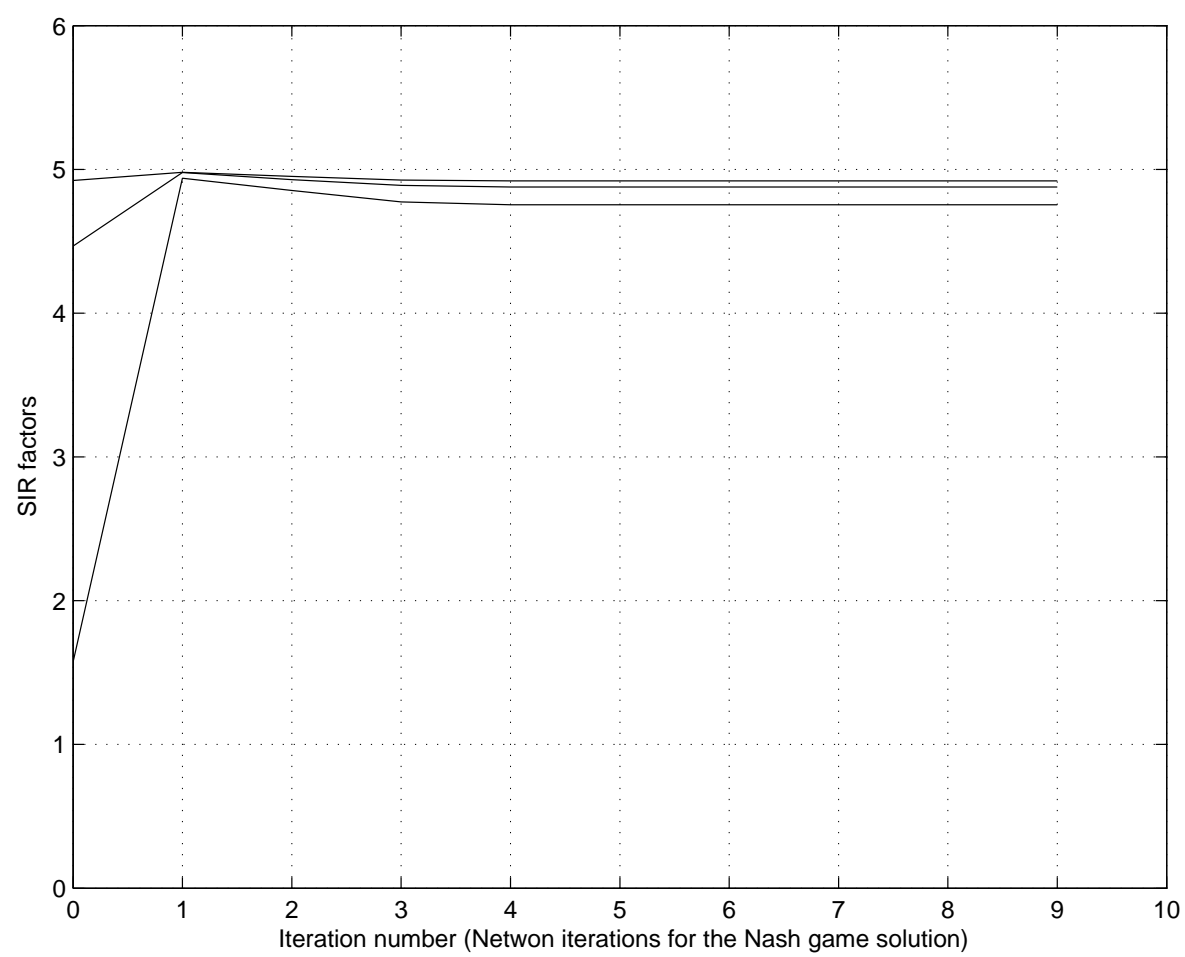

Figure 4. SIR's computed for the three mobiles using fixed-point iterations. 


\section{REFERENCES}

1. J. M. Aein, "Power balancing in systems employing frequency reuse," COMSAT Technical Review 3(2), pp. 277-299, 1973.

2. H. J. Meyerhoff, "Method for computing the optimum power balance in multibeam satellites," COMSAT Technical Review 4(1), pp. 139-146, 1974.

3. R. W. Nettleton and H. Alavi, "Power control for a spread spectrum cellular mobile radio system," in Proceedings, IEEE Vehicular Technology Conference, pp. 242-246, 1983.

4. J. Zander, "Performance of optimum transmitter power control in cellular radio systems," IEEE Trans. Vehicular Technology 41, pp. 57-62, Feb. 1992.

5. J. Zander, "Distributed cochannel interference control in cellular radio systems," IEEE Trans. Vehicular Technology 41, pp. 305-311, Aug. 1992.

6. T. Alpcan, T. Basar, R. Srikant, and E. Atman, "CDMA uplink power control as a noncooperative game," in Proceedings of the 40th IEEE Conference on Decision and Control, 1, pp. 197-202, 2001.

7. H. Ji and C.-Y. Huang, "Non-cooperative uplink power control in cellular radio systems," Wireless Networks 4(3), pp. 233-240, 1998.

8. A. B. MacKenzie and S. B. Wicker, "Game theory in communications: Motivation, explanation, and application to power control," in Proceedings, IEEE GLOBECOM '01, pp. 821-826, 2001.

9. S. Koskie, Contributions to Dynamic Nash Games and Applications to Power Control for Wireless Networks. PhD thesis, Rutgers University, Jan. 2003.

10. T. Başar and G. J. Olsder, Dynamic Noncooperative Game Theory, SIAM, Philadelphia, 2nd ed., 1999.

11. J. Nash, "Noncooperative games," The Annals of Mathematics, 2nd ser. 54, pp. 286-295, 1951.

12. J. M. Ortega and W. C. Rheinboldt, Iterative Solution of Nonlinear Equations in Several Variables, Academic Press, New York, 1970.

13. R. L. Burden, J. D. Faires, and A. C. Reynolds, Numerical Analysis, Prindle, Weber \& Schmidt, Boston, 1981. 